\title{
The Genetics Corner: A Mother and Child with Cleft lip and Palate Have an Atypical 1p36 Deletion that Disrupts KIF1B, a Cause of Autosomal Dominant Charcot-Marie-Tooth Disease, Type 2A1
}

\section{Subhadra Ramanathan, MSc MS, Robin Dawn Clark, MD}

Case History:

An eight-week-old male infant with prenatally diagnosed bilateral cleft lip and palate was initially evaluated by the genetics team during a Craniofacial Team clinic appointment. His mother reported that prenatal care was intermittent, and the pregnancy was stressful due to emotional conflict with her partner. The baby was delivered by NSVD at term to a 42-year-old G2P1 $\rightarrow 2$ woman at a local community hospital. The baby was in the NICU for 12 days for feeding issues related to the oral cleft. BW was $2454 \mathrm{~g} \mathrm{(Z}$ score -2.01), discharge weight was $2531 \mathrm{~g}$. By eight weeks of age, he failed to thrive, and his weight was low at $2060 \mathrm{~g}$ ( $Z$ score -4.58). He had a poor suck, and there was concern that the formula had not been mixed appropriately at home. After his cleft lip and palate repair at eight months, his growth improved. His last recorded weight at 12 months was $9.48 \mathrm{~kg}$ (Z score -0.27).

"By eight weeks of age, he failed to thrive, and his weight was low at $2060 \mathrm{~g}$ (Z score -4.58). He had a poor suck, and there was concern that the formula had not been mixed appropriately at home. After his cleft lip and palate repair at eight months, his growth improved."
At eight weeks, he was developing appropriately. He smiled socially, laughed, and vocalized responsively. He regarded a face. $\mathrm{He}$ reached out for and mouthed objects. He moved all extremities symmetrically with good muscle tone and muscle mass.

The family history was significant for cleft lip in his mother, who had a repaired unilateral cleft lip and palate, right exotropia, and learning disabilities, although she was in regular classes. She did not graduate from high school. On examination, she had bilateral pes cavus and extensive, thick calluses on the plantar surfaces of both feet, suggesting a sensory neuropathy. The mother reported that her sister has a possible diagnosis of hereditary sensory and motor neuropathy (Charcot-Marie-Tooth disease or CMT). She had another sister who died at age 2 of heart problems.

"The mother reported that her sister has a possible diagnosis of hereditary sensory and motor neuropathy (CharcotMarie-Tooth disease or CMT). She had another sister who died at age 2 of heart problems."

Chromosome microarray analysis was ordered at this visit and revealed a 2.8 megabase interstitial deletion on chr1p36, subsequently confirmed to be maternally inherited: arr[hg19] $1 \mathrm{p} 36$. $22 p 36.21(10,388,064-13,182,587) \times 1$. The infant and his mother have an atypical interstitial $1 \mathrm{p} 36$ deletion that interrupts KIF1B, a gene associated with autosomal dominant Charcot-Marie-Tooth
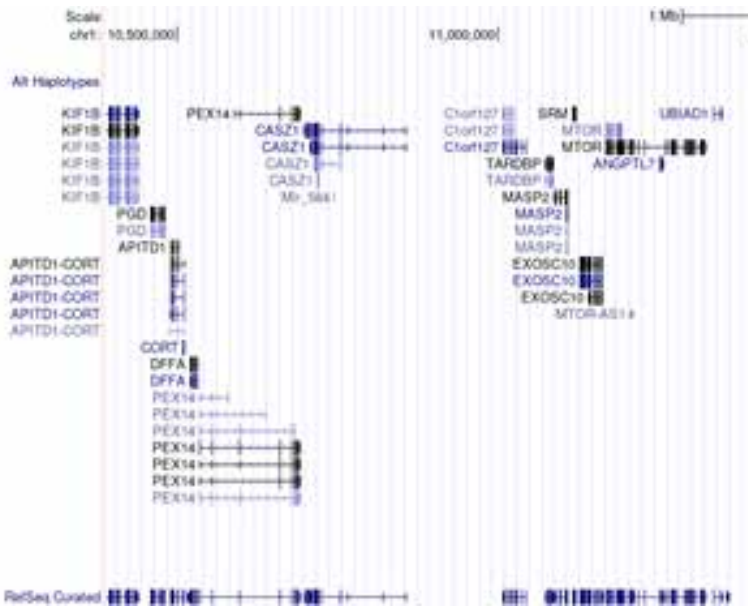

11.500,000/

29000000

130000001

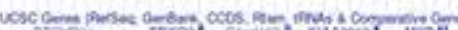

procente
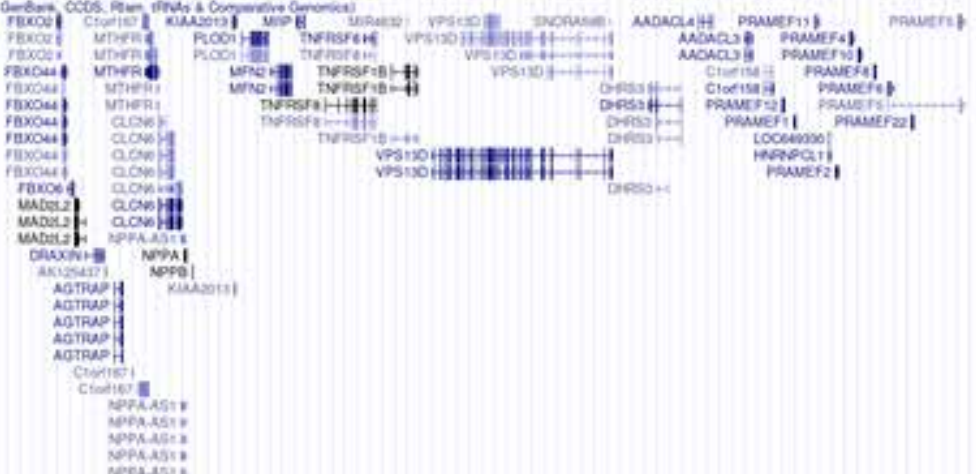

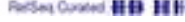

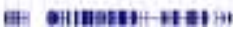

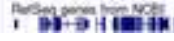

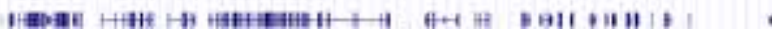

Figure 1: The genomic coordinates from the microarray report were used to identify the genes involved in this familial interstitial $1 p 36$ deletion. The genomic coordinates were inputted in the UCSC genome browser (https://genome.ucsc.edu), which shows that the distal breakpoint interrupts KIF1B (far left), a gene associated with autosomal dominant Charcot-Marie-Tooth disease (CMT). 


\section{Mitochondrion}

\section{ANTEROGRADE TRANSPORT}

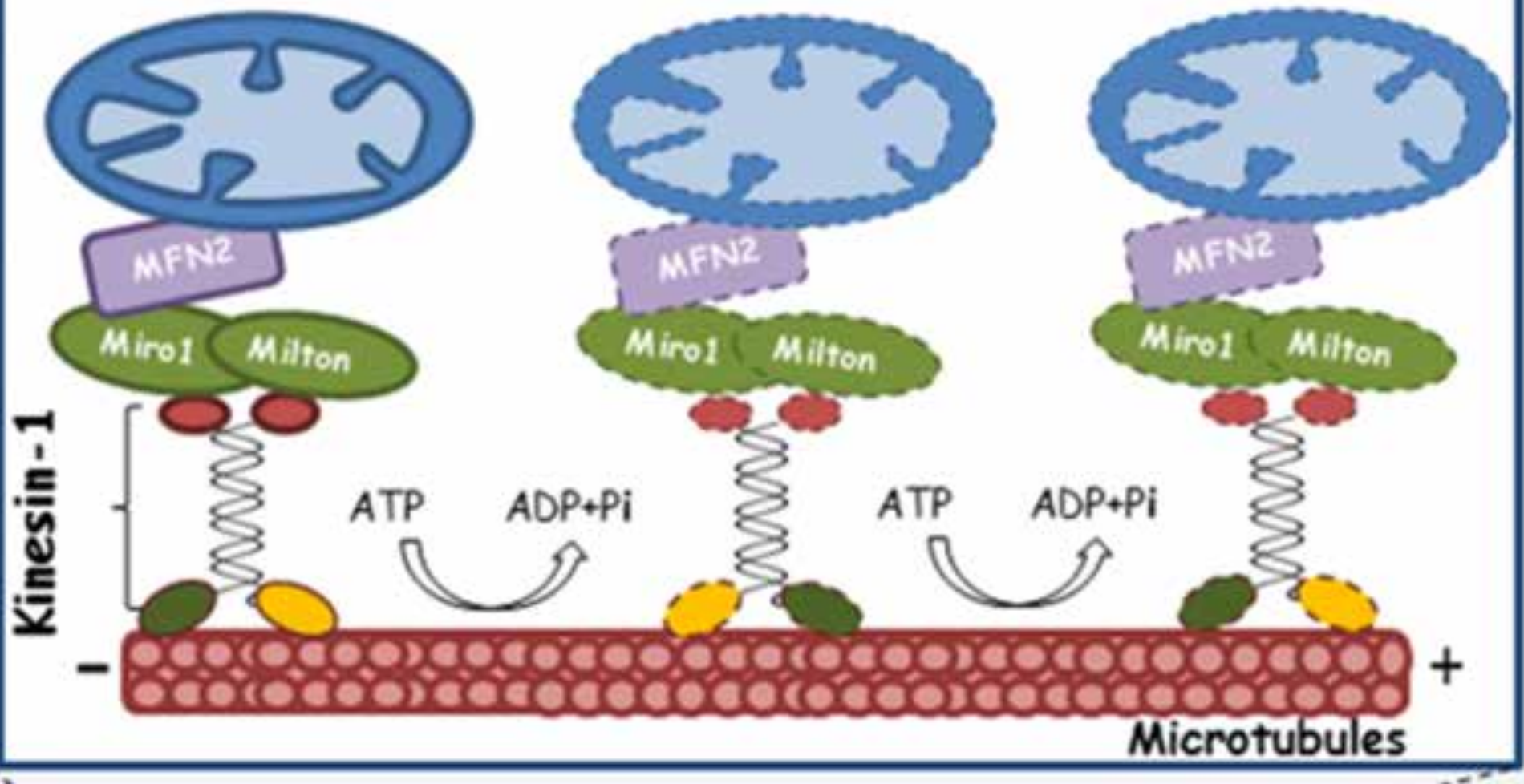

Figure 2: KIF1B encodes the kinesin-1 protein, a key component of a "molecular motor" that transports mitochondria in the peripheral nerve axon. This illustration shows how mitochondria are normally "walked" up the axon by coordinating the alternative binding and releasing of the two kinesin heads, keeping one head bound to the microtubular pathway at all times. Most kinesins move toward the plus end of the microtubular pathway. (Adapted from Pareyson, et al. 2015.) (2)

disease, type 2A1 (Figure 1). The mother underwent gene sequencing and deletion/duplication analysis for KIF1B (Invitae) that confirmed a large deletion in one homologue. She has been referred for a neurological assessment at a neuromuscular disease clinic.

\section{Assessment:}

The $1 \mathrm{p} 36$ deletion syndrome (OMIM 607872) occurs in 1 in 5000 newborns and is one of the most commonly reported chromosome deletion syndromes. Most are pure terminal deletions, and a smaller number are caused by interstitial deletions of varying size and position (10-29\%). Mosaicism and unbalanced translocations have been reported less commonly. There is wide clinical variability in chromosome $1 \mathrm{p} 36$ deletion syndrome, as the deletions vary in size, up to $30 \mathrm{Mb}$ of DNA. There is no single common breakpoint.

The classic 1 p36 deletion syndrome can be recognized by characteristic facial features: straight eyebrows, deep-set eyes, a wide nasal bridge with a pointed chin, and posteriorly-rotated, low-set, abnormal ears. Affected individuals have late-closing anterior fontanel, microcephaly, microbrachycephaly, and, often, seizures (44\%-58\%). Brain anomalies, orofacial clefting, congenital heart defects, cardiomyopathy, usually left ventricular noncompaction type, renal anomalies, and sensorineural hearing impairment are common. The region on $1 p 36$ that is responsible for cleft lip and palate has not been delineated. The areas on 1 p36 that are responsible for dysmorphic features and intellectual disability have been narrowed to 2 critical regions. (1)

Our patient's $2.8 \mathrm{Mb}$ deletion is smaller and more proximal than the more typical and generally larger classic, terminal 1 p36 microdeletions. This may help define and narrow the critical region associated with oral clefting in this syndrome. It is not clear at this time whether the maternal aunt who has CMT also has the familial chromosome 1p36 deletion, but she does not have an oral cleft. Our patient and his mother do not have hypotonia or dysmorphic facial features described in the classic 1 p36 deletion syndrome. Our patient has bilateral cleft lip and palate and hypertelorism but none of the other classic facial features. His echocardiogram

The only worldwide monthly publication
exclusively serving Pediatric and Adult
Cardiologists that focus on Congenital/
$\begin{aligned} & \text { CONGENITAL } \\ & \text { CARDIOLOGY } \\ & \text { TORAY }\end{aligned}$
Cardiothoral Heart Disease (CHD), and




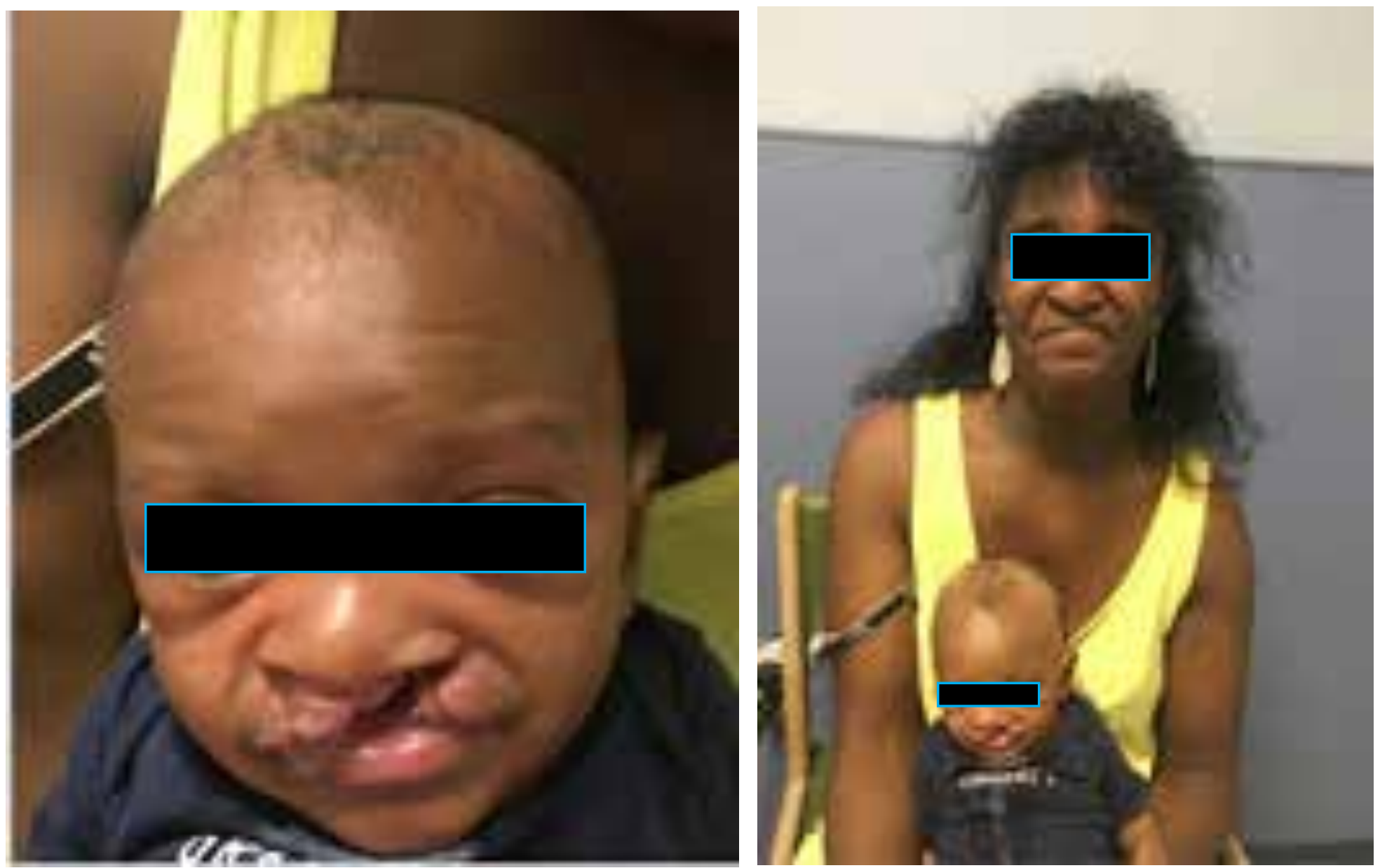

Figure 3: Bilateral cleft lip and palate, hypertelorism and infraorbital creases in the child and repaired unilateral cleft lip in his mother.

was normal. He had failure to thrive but has been gaining weight steadily since his surgical repair.

Charcot-Marie-Tooth disease (CMT) is divided into type 1 , the demyelinating form, characterized by a slow motor median nerve conduction velocity (NCV) (less than $38 \mathrm{~m} / \mathrm{s}$ ), and type 2, the axonal form, with a normal or slightly reduced NCV.

The energy requirements of peripheral nerve axons depend on the proper function and distribution of mitochondria. Abnormal mitochondrial transport along the axonal microtubular system can present as peripheral neuropathy and Charcot-Marie-Tooth disease. KIF1B (OMIM 605995, hg19 chr1:10,270,764-10,441,661) encodes a kinesin family motor protein, kinesin-1, that acts as the main anterograde motor in axons and binds mitochondria through adaptor proteins (Figure 2). Heterozygous pathogenic variants in KIF1B cause Charcot-Marie Tooth Disease axonal type 2A1, CMT2A1 (OMIM 118210).

Although the loss of the KIF1B gene or haploinsufficiency alone does not cause CMT2A1 and CMT has not been previously described in the 1 p36 deletion syndrome phenotype, (3) we postulate that in this case, disruption of KIF1B produces an abnormal gene product that interrupts normal axonal transport of mitochondria and causes CMT2A1. The mother has suggestive clinical findings of CMT; she has not yet undergone formal neurologic assessment and confirmatory diagnosis. If the diagnosis of CMT2A1 is confirmed in her, we anticipate that our patient will also exhibit signs of Charcot-Marie-Tooth disease, type 2A1, with time.

\section{Practical Applications:}

1. Carefully examine the parents of infants with congenital anomalies and take a detailed family history. In this case, the family history of an aunt with CMT leads us to examine the mother more thoroughly.

2. Use chromosome microarray as a first-line diagnostic test. Suspect a microdeletion or microduplication when intellectual disability, learning problems, delayed development, or congenital anomalies are seen in the parent of an affected child.

3. Recognize that 1 p36 microdeletion syndrome is a relatively common and variable microdeletion syndrome, including cleft lip and palate.

4. Input genomic coordinates at https://genome.ucsc.edu to identify genes in the chromosome segment of interest and pay attention to genes at the breakpoints that the deletion or duplication may disrupt.

\section{References:}

1. Shimada S, Shimojima K, Okamoto N, et al. Microarray analysis of 50 patients reveals the critical chromosomal regions responsible for $1 p 36$ deletion syndrome-related complications. Brain Dev. 2015 May;37(5):515-26. PMID: 25172301.

2. Pareyson D, Saveri P, Sagnelli A, Piscosquito G. Mitochondrial dynamics and inherited peripheral nerve diseases. Neurosci Lett. 2015 Jun 2;596:66-77. PMID: 
25847151.

3. Xu F, Takahashi H, Tanaka Y, et al. KIF1B $\beta$ mutations detected in hereditary neuropathy impair IGF1R transport and axon growth. J Cell Biol. 2018 Oct 1;217(10):34803496. PMID: 30126838

Disclosures: The authors have no relevant disclosures.

\section{NT}

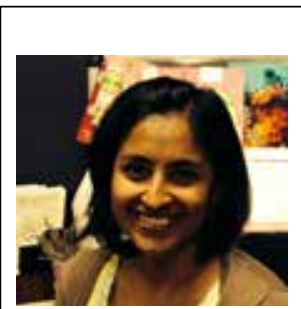

Subhadra (Subha) Ramanathan, M.Sc., M.S. Licensed and Certified Genetic Counselor Assistant Professor, Pediatrics Loma Linda University Health 2195 Club Center Drive, Ste A San Bernardino, CA 92408 Email: SRamanathan@llu.edu
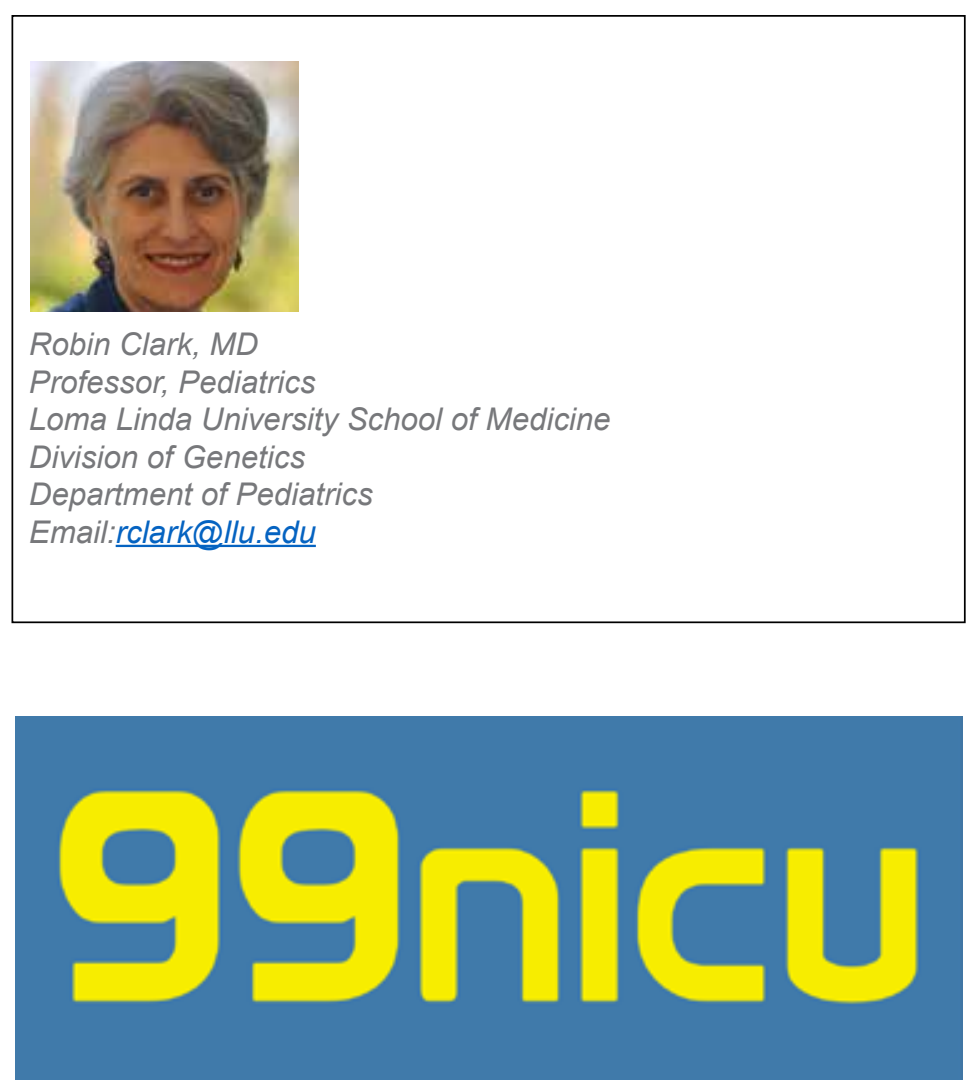

\section{Education that is...}

- Story-Driven

- Trauma-Informed

- Evidence-Based

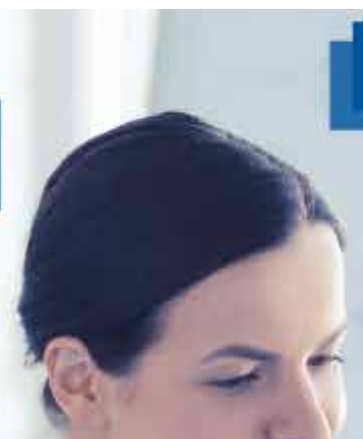

\section{Visit us at www.mynicunetwork.org}

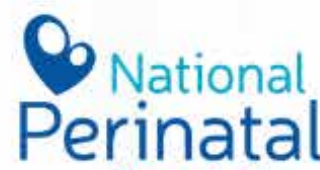
Association

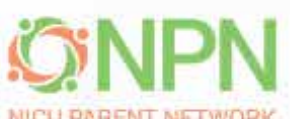

NICU PAFENT NETWORK

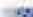
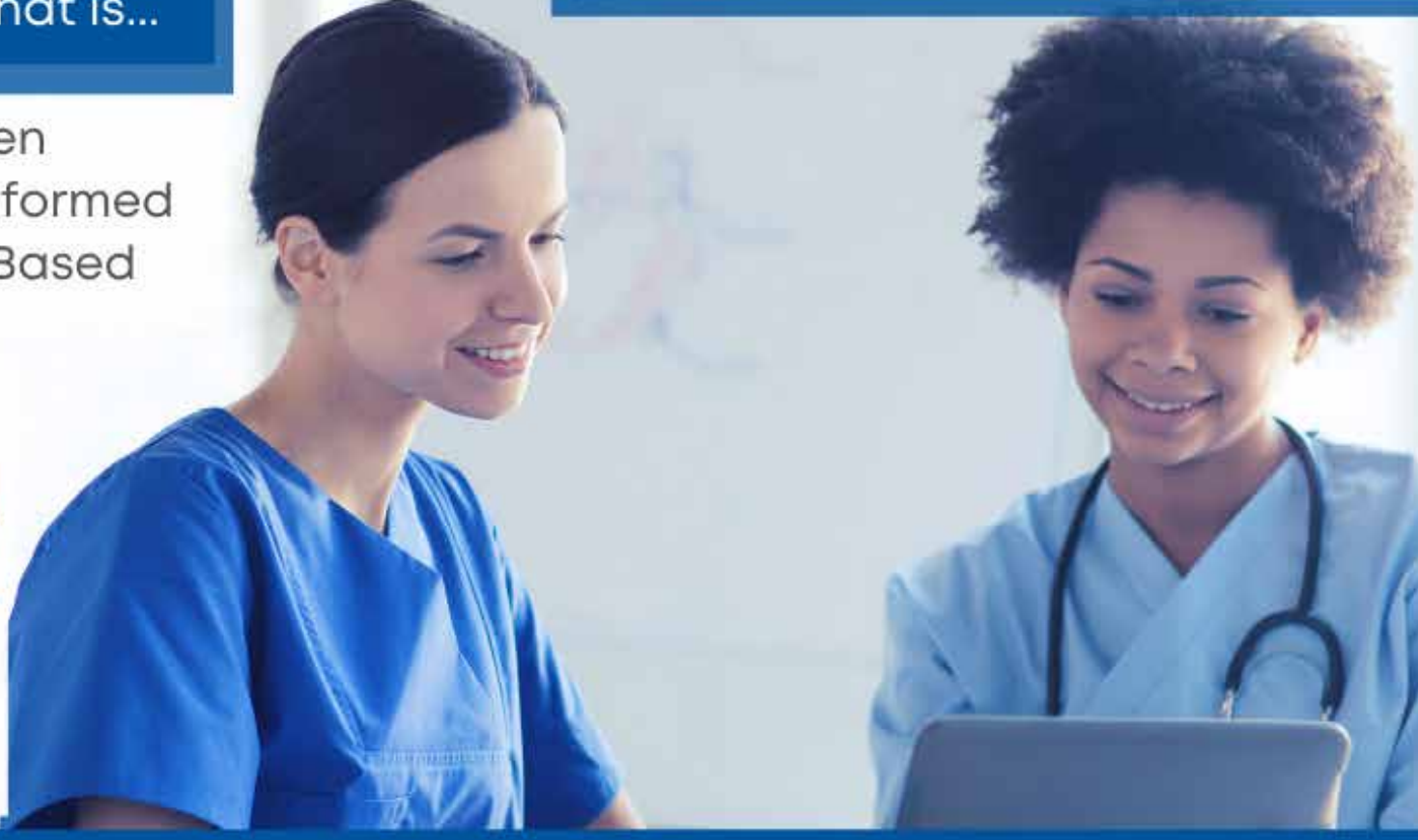

The preeminent provider of compelling perinatal education on

psychosocial support created through interprofessional collaboration 